\section{Which tonometry in eyes with keratoconus?}

H Altinkaynak ${ }^{1}$, C Kocasarac ${ }^{2}, \mathrm{H}_{\text {Dundar }}^{3}$, N Sayin ${ }^{4}$, N Kara $^{5}$, E Bozkurt ${ }^{6}$ and N Duru ${ }^{1}$

\begin{abstract}
Aims To compare intraocular pressure (IOP) measurements obtained with Goldmann applanation tonometery (GAT), dynamic contour tonometry (DCT), tonopen (TP), and ocular response analyzer (ORA), and to determine the influence of Amsler grade and central corneal thickness (CCT) on the IOP readings in eyes with keratoconus that are classified into four groups according to the Amsler - Krumeich classification.

Methods All eyes with keratoconus were separated into four groups using Amsler - Krumeich classification for keratoconus. IOP was measured in 202 eyes of 202 patients with keratoconus using GAT, DCT, TP, and ORA.

Results The IOP differences revealed no significant difference among the Amsler degree in the DCT and corneal-compensated IOP (IOPcc) measurements ( $P>0.05$ for all).

There was no statistically significant difference in terms of IOP differences between GAT and IOPcc $(P>0.05)$, TP and Goldmann-correlated measure of IOP (IOPg; $P>0.05)$ in the Amsler I, while the IOP measurements revealed significant difference among the measurements of the four different tonometers in the Amsler II, Amsler III, and Amsler IV $(P<\mathbf{0 . 0 5}$ for all). Conclusions There was no significant association between DCT IOP or IOPcc and CCT in eyes with keratoconus; no statistically significant difference was found between keratoconus stages and the control group in terms of the IOP analyzed with these two techniques. These two techniques may be the most stable in the measurement of IOP in different keratoconus stages. However, no IOP technique can be used interchangeably with other techniques in the follow-up of keratoconus patients.
\end{abstract}

Eye (2016) 30, 431-437; doi:10.1038/eye.2015.248; published online 4 December 2015

\section{Introduction}

Keratoconus is ectatic corneal disorder characterized by progressive corneal thinning and protrusion. ${ }^{1}$ Intraocular pressure (IOP) is an important parameter in the diagnosis and management of glaucoma. Obtaining an accurate IOP measurement is a greater challenge in keratoconic eyes. ${ }^{2-4}$ Axial stromal thinning, progressive conical distortion with irregular astigmatism and apical protrusion make accurate measurement of IOP problematic. 5,6

Goldmann applanation tonometry (GAT) is still the gold standard for IOP measurement in the clinical management of glaucoma, although measurement errors due to central corneal thickness (CCT), axial length, and corneal curvature are known. ${ }^{7-9}$ The ocular response analyzer (ORA) was designed to provide IOP assessments that are independent of CCT. The instrument provides a corneal hysteresis $(\mathrm{CH})$, a corneal resistance factor (CRF), a Goldmanncorrelated measure of IOP (IOPg) and a corneal-compensated IOP (IOPcc). ${ }^{10,11}$ Dynamic contour tonometry (DCT) uses an applanating probe that nearly matches the corneal surface contour with a piezoelectrical device for pressure measurement attached to its apex. Pressure on both sides of the cornea is equalized as the cornea takes the tip contour, and a pressuresensitive area in the center of the contour surface with a built-in microprocessor provides a direct and continuous transcorneal measurement of IOP that is independent of corneal properties. ${ }^{12-15}$ Mackay - Marg-type tonopens (TP) are often used tonometers owing to how easy they are to carry and use. During the straightening of the cornea by the flat base on the tip of the TP, a tightening meter creates an electrical impulse. A microprocessor senses the suitable power curves. It calculates the mean of 4-10 measurements and forms a last digital output with the percentages of variability. ${ }^{16,17}$

There are various studies in the literature that compare IOP in keratoconic eyes. ${ }^{18-21}$ It is still debated that tonometer provides the most accurate IOP measurement. The purpose of this study was to identify the agreement between IOP readings obtained by these four tonometers (GAT, DCT, TP, and ORA) and to determine the



${ }^{1}$ Department of Ophthalmology, Ankara Ataturk Education and Research Hospital, Yıldirim Beyazit University, Ankara, Turkey

${ }^{2}$ Department of Ophthalmology, Kelkit

State Hospital,

Gümüşhane, Turkey

${ }^{3}$ Department of Ophthalmology, Selahaddin Eyyubi State Hospital, Diyarbakır, Turkey

${ }^{4}$ Department of Ophthalmology, Kanuni Sultan Suleyman Education and Research Hospital, Istanbul, Turkey

${ }^{5}$ Department of Ophthalmology, Gaziantep University School of Medicine, Gaziantep, Turkey

${ }^{6}$ Department of Ophthalmology, Beyoğlu Eye Education and Research Hospital, istanbul, Turkey

Correspondence:

H Altinkaynak, Department of Ophthalmology, Ankara Ataturk Education and Research Hospital, Yıldirim Beyazit University, Ankara, 06560, Turkey

Tel: +05063509748

Fax: +90 3122912525 . E-mail: altinkaynak167@ yahoo.com

Received: 11 January 2015 Accepted in revised form: 20 October 2015

Published online: 4 December 2015 
influence of Amsler grade and CCT on the IOP readings in eyes with keratoconus.

\section{Materials and methods}

\section{Study population and design}

This prospective and interventional study was performed at the Istanbul Beyoglu Eye Education and Research Hospital. The study followed the tenets of the Declaration of Helsinki and was approved by the local ethics committee. All participants received oral and written information about the study, and each participant provided written informed consent.

Two hundred and two eyes of 202 patients with keratoconus were evaluated from a specialist corneal clinic. The control group was formed with 49 eyes of 49 healthy subjects who were matched with patients in the study group for mean age. The control group was examined in same corneal clinic and excluded with respect to keratoconus.

Each participant underwent a comprehensive ophthalmologic examination, including review of medical history, corrected distance visual acuity, slit-lamp microscopy, and funduscopic examination. Axial length measurements with the IOL Master 500 (Carl Zeiss Meditec Inc., Jena, Germany). Exclusion criteria were subjects with active ocular inflammation, corneal epithelial defects, stromal opacity or scarring, glaucoma, known systemic illnesses, chronic use of topical ocular medications, a history of any other structural corneal disease than keratoconus or previously performed ophthalmological surgery of any kind. The participants of the groups were asked not to wear contact lenses at least 1 week before evaluation.

The keratoconus diagnosis was defined by clinical examination (slit-lamp biomicroscopy signs such as Fleisher ring, corneal thinning, Vogt striae, and enlarged corneal nerves, scissoring reflex on retinoscopy and characteristic external clinical findings such as Rizzuti and Munson signs) and confirmed by Scheimpflug camera with a Placido disk topographer (Sirius; Costruzione Strumenti Oftalmici, Florence, Italy). The diagnosis of keratoconus was made by an experienced corneal specialist (EB). CCT was also detected by Sirius. ${ }^{18}$ Sirius was performed before recording IOP to rule out any error induced by probe contact. All eyes with keratoconus were separated into four groups using Amsler-Krumeich classification for keratoconus. ${ }^{22}$ IOP was measured always in the same order, ORA, GAT, DCT, and TP, to avoid IOP reduction by GAT, DCT, and TP. All measurements were performed by the same clinician (HA). Attempting to minimize bias of diurnal IOP variations, we conducted all measurements between 0900 and 1100 hours. Each measurement was made three times, with an interval between each measurement of $15 \mathrm{~min}$.

\section{Measurements}

Initially, each subject underwent assessment with ORA (Reichert Ophthalmic Instruments, Buffalo, NY, USA) that included measurement of IOPg, IOPcc, $\mathrm{CRF}$, and $\mathrm{CH}$. Three ORA measurements were performed in all patients by an experienced clinician (HA). Three good quality (symmetric, well-defined inward and outward applanation spike height) measurements were obtained for each eye. After the ORA measurements, applanation tonometry was performed with a Goldmann tonometer after a drop of proparakain hidroklorür $(0.05 \%)$ and a drop of fluorescein sodium $(0.25 \%)$ was instilled. To obtain accuracy of the GAT (Haag Streit, Koeniz, Switzerland) measurements in eyes with an astigmatism of 3 diopters, the glass cone was rotated to coincide with the middle of the cornea's 2 principle axes. After the GAT readings, IOP was measured with DCT (SMT Swiss Microtechnology, Port, Switzerland). The DCT is mounted on the slit-lamp similar to that used in the GAT readings and provides an absolute numerical output of IOP after coming in contact with the cornea for about $8 \mathrm{~s}$. A ' $\mathrm{Q}$ ' value is also displayed with the DCT measurements; it refers to the quality of data obtained. The ' $Q$ ' value is graded from 1 to 5 (Q 1 , the best; Q 5, the worst). In this study, three consecutive DCT measurements were performed in all eyes. The only measurements of a quality of one or two were recorded.

Finally, IOP was measured with the TP (Medtronic Solan Tono-Pen XL Applanation Tonometer, Jacksonville, FL, USA) calibrated for each eye. Before each measurement, a sterile cover (Ocu-Film Tono-Pen Tip Covers Medtronic Ophthalmics, USA) was placed on the tip of the TP. The TP was prepared to measure by pressing the 'open' button once, and the measurement was made by slowly touching the TP to the peak point of the cornea to be measured following the application of a topical anesthesia. The means of three measurements that did not have more than $3 \mathrm{~mm} \mathrm{Hg}$ of difference were taken and recorded.

\section{Statistical analysis}

All statistical tests were performed using SPSS, version 16 (Statistical Package for the Social Sciences, SPSS Inc., Chicago, IL, USA). Measurements of the one eye of each participant were used for analyses. Data normality was confirmed using Kolmogorov-Smirnov test $(P>0.05)$, and a paired $t$-test was used to compare variables between the subgroups composed according to Amsler-Krumeich classification for keratoconus and control group. 
ANOVA test was used for parametric comparisons between subgroups of more than two, and the significance between groups was determined by Bonferroni test. Pearson correlations were used to assess the dependence of the tonometers on CCT. Statistical significance was defined as a $P$-value of 0.05 .

\section{Results}

Table 1 shows the baseline characteristics in the four subgroups composed according to Amsler-Krumeich classification for keratoconus and control groups. Two hundred and two eyes of 202 patients with keratoconus (99 males, 103 females) and 49 eyes of 49 normal subjects were included. Amsler I had 52, Amsler II had 50, Amsler III had 52, and Amsler IV had 48 patients with keratoconus according to Amsler - Krumeich classification. There was a statistically significant difference of average simulated keratometry (mean sim K) and CCT measurements among the four subgroups and control group $(P<0.001)$. Mean IOP measurements obtained with the GAT, DCT, TP, and ORA in the four subgroups composed according to Amsler-Krumeich classification for keratoconus and control group are shown in Table 2.
IOP difference in the GAT measurements between group of Amsler I and Amsler IV, Amsler II and Amsler IV, Amsler III and control, Amsler IV and control were statistically significantly different $(P<0.05$ for all). There was a statistically significant difference in terms of IOP differences between Amsler I and Amsler IV, Amsler II and Amsler IV, Amsler II and control, Amsler III and control, Amsler IV and control in the TP measurements $(P<0.05$ for all $)$. Also, there was a statistically significant difference in terms of IOP differences between Amsler I and Amsler II, Amsler I and Amsler III, Amsler I and Amsler IV, Amsler II and Amsler IV, Amsler II and Control, Amsler III and Amsler IV, Amsler III and Control, Amsler IV and Control in the IOPg measurements $(P<0.05$ for all) while the IOP differences revealed no significant difference among the Amsler degree in the DCT and IOPCC measurements $(P>0.05$ for all).

The differences between the measurements of the four different tonometers in the four subgroups composed according to Amsler-Krumeich classification for keratoconus and control group are shown in Table 3 and Table 4, which shows the correlation for the keratoconic subgroups and control group between CCT and GAT, DCT, TP, IOPg, IOPcc. The CCT value was significantly associated with mean IOPg in the keratoconic subgroups.

Table 1 Basic demographic data of the four subgroups composed according to Amsler - Krumeich classification for keratoconus and control group

\begin{tabular}{|c|c|c|c|c|c|}
\hline & \multicolumn{5}{|c|}{ Amsler degree and control group } \\
\hline & Amsler I & Amsler II & Amsler III & Amsler IV & Control group \\
\hline Number of eyes/patients & $52 / 52$ & $50 / 50$ & $52 / 52$ & $48 / 48$ & $49 / 49$ \\
\hline Gender male/female & $28 / 24$ & $23 / 27$ & $25 / 27$ & $23 / 25$ & $24 / 25$ \\
\hline $\begin{array}{l}\text { Age, years mean } \pm S D \text {, } \\
\text { range }\end{array}$ & $\begin{array}{c}27.6 \pm 7.2 \\
21-30\end{array}$ & $\begin{array}{c}23.6 \pm 5.5 \\
19-29\end{array}$ & $\begin{array}{c}28.3 \pm 6.3 \\
21-32\end{array}$ & $\begin{array}{c}26.7 \pm 9.3 \\
19-32\end{array}$ & $\begin{array}{c}25.7 \pm 5.8 \\
20-30\end{array}$ \\
\hline $\begin{array}{l}\text { Mean sim } \mathrm{K}, \mathrm{D} \text { mean } \pm \mathrm{SD} \text {, } \\
\text { range }\end{array}$ & $\begin{array}{c}47.6 \pm 1.18 \\
45-48\end{array}$ & $\begin{array}{c}50.4 \pm 1.76 \\
48-53\end{array}$ & $\begin{array}{l}54.8 \pm 1.11 \\
53-55\end{array}$ & $\begin{array}{c}57.4 \pm 3.82 \\
55-69.2\end{array}$ & $\begin{array}{c}44.37 \pm 1.81 \\
40.9-45\end{array}$ \\
\hline $\mathrm{CCT}, \mu \mathrm{m}$ mean $\pm \mathrm{SD}$ & $475.4 \pm 44.2$ & $460.3 \pm 23.9$ & $455.2 \pm 24.4$ & $426.3 \pm 51.1$ & $531.1 \pm 40.6$ \\
\hline
\end{tabular}

Abbreviations: CCT, central corneal thickness; D, diopters; Mean sim K, average simulated keratometry.

Table 2 Mean IOP measurements obtained with the GAT, DCT, TP, and ORA in the four subgroups composed according to Amsler - Krumeich classification for keratoconus and control group

\begin{tabular}{lccccc}
\hline & \multicolumn{3}{c}{ Amsler degree and control group } \\
\cline { 2 - 6 } & Amsler I & Amsler II & Amsler III & Amsler IV & Control group \\
\hline ORA mean $\pm S D,(m m ~ H g)$ & & & & & \\
IOPg & $11.71 \pm 3.08$ & $9.71 \pm 2.75$ & $9.02 \pm 3.50$ & $6.82 \pm 2.64$ & $13.20 \pm 5.83$ \\
IOPcc & $13.87 \pm 2.32$ & $13.89 \pm 2.45$ & $13.73 \pm 2.36$ & $13.22 \pm 2.46$ & $13.30 \pm 5.54$ \\
GAT mean \pm SD, $(\mathrm{mm} \mathrm{Hg})$ & $13.76 \pm 4.22$ & $12.79 \pm 2.43$ & $12.29 \pm 2.95$ & $11.12 \pm 3.70$ & $14.19 \pm 4.90$ \\
DKT mean \pm SD, $(\mathrm{mm} \mathrm{Hg})$ & $16.66 \pm 2.91$ & $15.97 \pm 2.57$ & $17.03 \pm 2.85$ & $15.94 \pm 2.65$ & $17.52 \pm 3.84$ \\
TP mean \pm SD, $(\mathrm{mm} \mathrm{Hg})$ & $11.51 \pm 2.99$ & $11.09 \pm 2.47$ & $10.33 \pm 2.85$ & $9.24 \pm 2.80$ & $13.34 \pm 4.64$ \\
\hline
\end{tabular}

Abbreviations: DCT, dynamic contour tonometry; GAT, Goldmann applanation tonometer; IOP, intraocular pressure; IOPcc, corneal-compensated IOP; IOPg, Goldmann-correlated IOP value; ORA, ocular response analyzer; TP, tonopen. 
Table 3 The differences between the measurements of the four different tonometers in the four subgroups composed according to Amsler - Krumeich classification for keratoconus and control group

\begin{tabular}{|c|c|c|c|c|c|}
\hline & \multicolumn{5}{|c|}{ Mean difference $($ mean $\pm S D)(m m H g)$} \\
\hline & Amsler I & Amsler II & Amsler III & Amsler IV & Control group \\
\hline \multicolumn{6}{|l|}{ GAT } \\
\hline $\mathrm{DCT}$ & $-2.90 \pm 3.95$ & $-3.25 \pm 2.61$ & $-4.74 \pm 3.53$ & $-4.82 \pm 3.58$ & $-3.34 \pm 3.04$ \\
\hline $\mathrm{TP}$ & $2.24 \pm 2.99$ & $1.69 \pm 2.96$ & $1.95 \pm 3.73$ & $1.87 \pm 2.86$ & $0.83 \pm 4.17$ \\
\hline $\mathrm{IOPg}$ & $2.04 \pm 3.69$ & $3.08 \pm 2.35$ & $3.25 \pm 3.46$ & $4.29 \pm 3.28$ & $0.98 \pm 2.24$ \\
\hline IOPcC & $-0.11 \pm 4.13$ & $-1.10 \pm 1.97$ & $-1.45 \pm 2.96$ & $-1.98 \pm 3.65$ & $0.88 \pm 3.03$ \\
\hline \multicolumn{6}{|l|}{$D C T$} \\
\hline GAT & $2.90 \pm 3.95$ & $3.25 \pm 2.61$ & $4.74 \pm 3.53$ & $4.82 \pm 3.58$ & $3.34 \pm 3.04$ \\
\hline $\mathrm{TP}$ & $5.15 \pm 2.83$ & $4.95 \pm 2.63$ & $6.70 \pm 3.79$ & $6.70 \pm 2.82$ & $4.17 \pm 3.97$ \\
\hline $\mathrm{IOPg}$ & $4.94 \pm 3.33$ & $6.33 \pm 2.68$ & $8.00 \pm 3.11$ & $9.12 \pm 3.07$ & $4.32 \pm 4.05$ \\
\hline IOPcc & $2.78 \pm 3.37$ & $2.08 \pm 2.51$ & $3.29 \pm 2.53$ & $2.84 \pm 3.82$ & $4.22 \pm 4.11$ \\
\hline \multicolumn{6}{|l|}{ TP } \\
\hline GAT & $-2.24 \pm 2.99$ & $-1.69 \pm 2.96$ & $-1.95 \pm 3.73$ & $-1.87 \pm 2.86$ & $0.83 \pm 4.17$ \\
\hline $\mathrm{DCT}$ & $-5.15 \pm 2.83$ & $-4.95 \pm 2.63$ & $-6.70 \pm 3.79$ & $-6.70 \pm 2.82$ & $-4.17 \pm 3.97$ \\
\hline IOPg & $-0.20 \pm 2.79$ & $1.38 \pm 2.25$ & $1.30 \pm 3.85$ & $2.41 \pm 2.40$ & $0.14 \pm 4.19$ \\
\hline IOPcC & $-2.36 \pm 3.18$ & $-2.80 \pm 2.38$ & $-3.40 \pm 3.35$ & $-3.86 \pm 2.79$ & $0.04 \pm 4.12$ \\
\hline \multicolumn{6}{|l|}{$\mathrm{IOPg}$} \\
\hline GAT & $-2.04 \pm 3.69$ & $-3.08 \pm 2.35$ & $-3.25 \pm 3.46$ & $-4.29 \pm 3.28$ & $-0.98 \pm 2.24$ \\
\hline DCT & $-4.94 \pm 3.33$ & $-6.17 \pm 2.68$ & $-8.00 \pm 3.11$ & $-9.12 \pm 3.07$ & $-4.32 \pm 4.05$ \\
\hline TP & $0.20 \pm 2.79$ & $-1.38 \pm 2.25$ & $-1.30 \pm 3.85$ & $-2.41 \pm 2.40$ & $0.14 \pm 4.19$ \\
\hline IOPcC & $-2.16 \pm 1.71$ & $-4.18 \pm 1.41$ & $-4.70 \pm 1.99$ & $-6.27 \pm 1.95$ & $-0.1 \pm 2.22$ \\
\hline \multicolumn{6}{|l|}{$I O P C C$} \\
\hline GAT & $0.11 \pm 4.13$ & $1.10 \pm 1.97$ & $1.45 \pm 2.96$ & $1.98 \pm 3.65$ & $-0.88 \pm 3.03$ \\
\hline DCT & $-2.78 \pm 3.37$ & $-2.08 \pm 2.51$ & $-4.29 \pm 2.53$ & $-2.84 \pm 3.82$ & $-4.22 \pm 4.11$ \\
\hline $\mathrm{TP}$ & $2.36 \pm 3.18$ & $2.80 \pm 2.38$ & $2.40 \pm 3.35$ & $3.86 \pm 2.79$ & $0.04 \pm 4.12$ \\
\hline $\mathrm{IOPg}$ & $2.16 \pm 1.71$ & $4.18 \pm 1.41$ & $4.70 \pm 1.99$ & $6.27 \pm 1.95$ & $0.1 \pm 2.22$ \\
\hline
\end{tabular}

Abbreviations: DCT, dynamic contour tonometry; GAT, Goldmann applanation tonometer; IOPcc, corneal-compensated IOP; IOPg, Goldmann-correlated IOP value; TP, tonopen. Italic entries represent significant values, $P<0.05$, Bonferroni test for all.

\section{Discussion}

The easy, safe, and accurate measurement of IOP is important in the diagnosis and follow-up of patients with glaucoma. Obtaining an accurate IOP measurement has always been an important problem in keratoconus patients. It is difficult to have an accurate IOP measurement in keratoconus patients because of the decrease in corneal thickness, change in biomechanical features and deformation of the corneal surface. $2,3,23,24$

There are studies showing different results of the effect of CCT in IOP measurements of an eye with keratoconus. ${ }^{18-21,25}$ Mollan et al studied the association between IOP and CCT by using four tonometric methods (GAT, ORA, Pascal DCT, and TP) in 76 eyes with keratoconus. The results showed an association between IOPg, GAT, and CCT, while no association was found between DCT, TP, IOPCC, and CCT. The IOP measurement method that was least affected by CCT was Pascal DCT. ${ }^{4}$ Ozbek et al compared the IOP readings of the GAT, TP, and DCT. They found that unlike the GAT and TP, DCT was not associated with CCT in the keratoconus patients. Unterlauft et al compared DCT and GAT measurements in a study they conducted on 114 eyes with keratoconus. They found that both techniques were independent of CCT. ${ }^{21}$ Goldich et al compared ORA and GAT in a study they conducted on 59 eyes with keratoconus. In their study, they found that IOPcC and GAT were independent of CCT and that IOPg was significantly associated with CCT. ${ }^{20}$ In our study, no association was found between GAT, DCT, TP, IOPcc, and CCT in all the stages of keratoconus. On the other hand, a significant positive correlation was found between IOPg and CCT in all the stages of keratoconus. In the control group, an association was found between GAT, IOPg, TP, and CCT.

There are also studies with different results that compared the measurements of GAT, DCT, TP, IOPg, and IOPcc in eyes with keratoconus and examined the differences between the measurements. Goldich et al compared ORA and GAT in a study they conducted on 59 eyes with keratoconus. In their study, they found that the 
Table 4 The correlation for the keratoconic subgroups and control group between CCT and GAT, DCT, TP, IOPg, IOPcC

\begin{tabular}{|c|c|c|c|c|c|}
\hline & \multicolumn{5}{|c|}{ Central corneal thickness } \\
\hline & Amsler I & Amsler II & Amsler III & Amsler IV & Control group \\
\hline \multicolumn{6}{|l|}{ GAT } \\
\hline Pearson Correlation & 0.121 & 0.223 & 0.227 & 0.367 & 0.407 \\
\hline $\mathrm{P}$ & 0.565 & 0.263 & 0.321 & 0.231 & 0.035 \\
\hline \multicolumn{6}{|l|}{$D C T$} \\
\hline Pearson Correlation & 0.502 & 0.358 & 0.140 & 0.315 & 0.167 \\
\hline $\mathrm{P}$ & 0.061 & 0.066 & 0.544 & 0.070 & 0.406 \\
\hline \multicolumn{6}{|l|}{ TP } \\
\hline Pearson Correlation & -0.068 & 0.403 & -0.004 & 0.247 & 0.478 \\
\hline $\mathrm{P}$ & 0.747 & 0.145 & 0.987 & 0.159 & 0.012 \\
\hline \multicolumn{6}{|l|}{ IOPg } \\
\hline Pearson Correlation & 0.613 & 0.530 & 0.416 & 0.505 & 0.502 \\
\hline $\mathrm{P}$ & 0.005 & 0.004 & 0.040 & 0.002 & 0.008 \\
\hline \multicolumn{6}{|l|}{$I O P c C$} \\
\hline Pearson Correlation & -0.095 & 0.350 & 0.104 & 0.217 & 0.246 \\
\hline $\mathrm{P}$ & 0.651 & 0.074 & 0.653 & 0.217 & 0.216 \\
\hline
\end{tabular}

Abbreviations: CCT, central corneal thickness; DCT, dynamic contour tonometry; GAT, Goldmann applanation tonometer; IOPcc, corneal-compensated IOP; IOPg, Goldmann-correlated IOP value; TP, tonopen. Italic entries represent significant values, $P<0.05$.

IOP was $9.56 \pm 2.8 \mathrm{~mm} \mathrm{Hg}$ with $\mathrm{IOPg}, 13.3 \pm 2.5 \mathrm{~mm} \mathrm{Hg}$ with IOPcc, and $10.9 \pm 2.0 \mathrm{~mm} \mathrm{Hg}$ with GAT. They reported that the differences in mean IOP values between GAT and IOPg and between GAT and IOPcc were statistically significant and that these tonometers should not be used interchangeably. On the contrary, they should be used in a supplementary fashion to evaluate IOP in eyes with keratoconus. ${ }^{20}$ A study was conducted by Mollan et al with 118 healthy eyes and 76 eyes with keratoconus that compared four different tonometric methods (GAT, ORA, Pascal DCT, and TP). In all IOP measurement values, except for IOPg in the control group and IOPcc in the keratoconus group, the GAT measurements were lower when compared with other measurement techniques. Although they stated that TP was independent from all corneal parameters, they also pointed out that TP measurements were higher than those of GAT in the control and keratoconus groups, and thus it could not be the ideal measurement technique in the keratoconus group. They further stated that IOPcC and DCT measurements were independent of corneal parameters and their erroneous measurement rates were low; thus, they could be the ideal measurement technique in eyes with keratoconus. ${ }^{4}$

There is only one study in literature that compares IOP techniques in terms of keratoconus stages. In this study, Unterlauft et al grouped the DCT and GAT measurements of 114 keratoconus patients into 4 stages based on the Amsler - Krumeich keratoconus classification. They did not find any statistically significant difference between keratoconus stages in terms of IOP measured by DCT and GAT techniques. ${ }^{18}$ Also, they stated that GAT and DCT seem to be independent of CCT but cannot be used interchangeably when following up IOP in eyes with keratoconus. Our study has the broadest series in literature that compares IOP techniques in eyes with keratoconus based on the Amsler-Krumeich keratoconus classification. In our study, 202 eyes with keratoconus were grouped in four stages based on the Amsler-Krumeich keratoconus classification, and we examined the association between GAT, DCT, TP, IOPg, and IOPcc. According to the results, in IOP measurements by GAT, the highest IOP measurement was in Amsler I, while the lowest IOP measurement was in Amsler IV. We found that the IOP difference in the GAT measurements between the groups of Amsler I and Amsler IV, Amsler II and Amsler IV, Amsler III and the control, and Amsler IV and the control were significantly different statistically. This difference can result from a surface irregularity caused by a thin cornea, especially in advanced-stage keratoconus, high keratometric values and topographic dysregulation.

In our study, although there was no statistically significant association between GAT measurements and CCT, there were statistically significant IOP differences between the keratoconus stages and the lower IOP in the control group when compared with the keratoconus group, with GAT being affected by the line-of-vision and technical difficulties caused by surface irregularities, especially in advanced-stage keratoconus. The study also 
showed that GAT was not a suitable IOP measurement technique in eyes with keratoconus. In IOP measurements by DCT, no significant difference was found between keratoconus stages and the control group. In addition, there was no statistically significant association between DCT and CCT in any keratoconus stage or control group. Thus, DCT can be used as an ideal method in IOP measurement and follow-up in any stage of keratoconus. In IOP analysis with TP, the lowest IOP was in Amsler IV, while the highest IOP was in Amsler I. Although there was no statistically significant association between TP and CCT in eyes with keratoconus, statistically significant IOP differences between stages and the lower IOP in eyes with keratoconus when compared with the control group can show that TP is not a suitable IOP technique for keratoconus patients. In the IOPg measurements, the differences between Amsler stages and the IOPg value associated with CCT show us that the IOPg is not an ideal method in treating keratoconus. Finally, when IOPCC values in keratoconus patients were considered, no statistically significant differences were found between keratoconus stages and the control group in terms of the IOP value. At the same time, the results of our study showed that the IOPcc value is not associated with CCT. Owing to these features, an IOPcc value can be an ideal method in the IOP analysis of keratoconus patients.

Table 3 shows the data on the interchangeable use of IOP techniques in the IOP follow-up of keratoconus patients. According to the table, the differences between IOP techniques in Amsler II, Amsler III, and Amsler IV were found to be statistically significant, while in Amsler I the differences between GAT and IOPcc and between TP and IOPg were not found to be statistically significant. According to this data, no IOP method can be used interchangeably with keratoconus patients in the followup of IOP. We have tree limitation in this study. First, we evaluated only the effect of CCT on IOP, not effect $\mathrm{CRF}$ and $\mathrm{CH}$. CCT is only one aspect of the corneal biomechanics. Second, methodologic weakness was the nonmasked measurements with the GAT, DCT, TP, ORA. This could have introduced systematic bias. Third, In this study, the tonometers were used in the same order (ORA, GAT, DCT, and TP). GAT may result in lower IOP by DCT and TP. Therefore, the same order measurements cause a bias in the average IOP reading owing to applanation effects of GAT.

In conclusion, there was no significant association between DCT IOP or IOPCC and CCT in eyes with keratoconus; no statistically significant difference was found between keratoconus stages and the control group in terms of the IOP analyzed with these two techniques. Thus, these two techniques may be the most stable in the measurement of IOP in different keratoconus stages. However, no IOP technique can be used interchangeably with other techniques in the follow-up of eyes with keratoconus. Manometric studies may be helpful to determine the more precise and true IOP measurements in eyes with keratoconus in the future.

\section{Summary}

What was known before

- There was different results and the number of patients was small in other study.

- The grouping was not made according to Amsler - Krumeich classification for keratoconus.

What this study adds

- The grouping was made according to Amsler-Krumeich classification for keratoconus.

- The number of patients increased.

\section{Conflict of interest}

The authors declare no conflict of interest.

\section{References}

1 Rabinovitz YS. Keratoconus. Surv Ophthalmol 1998; 42: 297-319.

2 Shah S, Laiquzzaman M, Bhojwani R, Mantry S, Cunliffe I. Assessment of the biomechanical properties of the cornea with the ocular response analyzer in normal and keratoconic eyes. Invest Ophthalmol Vis Sci 2007; 48: 3026-3031.

3 Ortiz D, Pinero D, Shabayek MH, Arnalich-Montiel F, Alió JL. Corneal biomechanical properties in normal, post-laser in situ keratomileusis, and keratoconic eyes. J Cataract Refract Surg 2007; 33: 1371-1375.

4 Mollan SP, Wolffsohn JS, Nessim M, Laiquzzaman M, Sivakumar S, Hartley S et al. Accuracy of Goldmann, ocular response analyser, pascal and tonoPen $\mathrm{XL}$ tonometry in keratoconic and normal eyes. Br J Ophthalmol 2008; 92: 1661-1665.

5 Brooks AM, Robertson IF, Mahoney AM. Ocular rigidity and intraocular pressure in keratoconus. Aust J Ophthalmol 1984; 12: 317-324.

6 Bohm A, Kohlhaas M, Lerche RC, Bischoff B, Richard G. Measuring intraocular pressure in keratoconus. Effect of the changed biomechanics. Ophthalmologe 1997; 94: 771-774.

7 Herndon LW, Choudhri SA, Cox T, Damji KF, Shields MB, Allingham RR. Central corneal thickness in normal, glaucomatous, and ocular hypertensive eyes. Arch Ophthalmol 1997; 115: 1137-1141.

8 Herndon LW. Measuring intraocular pressure-adjustments for corneal thickness and new technologies. Curr Opin Ophthalmol 2006; 17: 115-119.

9 Whitacre MM, Stein RA, Hassanein K. The effect of corneal thickness on applanation tonometry. Am J Ophthalmol 1993; 115: 592-596

10 Wasielica-Poslednik J, Berisha F, Aliyeva S, Pfeiffer N, Hoffmann EM. Reproducibility of ocular response analyzer measurements and their correlation with central corneal thickness. Graefes Arch Clin Exp Ophthalmol 2010; 248: 1617-1622. 
11 Luce DA. Determining in vivo biomechanical properties of the cornea with an ocular response analyzer. J Cataract Refract Surg 2005; 31: 156-162.

12 Kniestedt C, Lin S, Choe J, Bostrom A, Nee M, Stamper RL. Clinical comparison of contour and applanation tonometry and their relationship to pachymetry. Arch Ophthalmol 2005; 123: 1532-1537.

13 Siganos DS, Papasterigiou GI, Moedas C. Assessment of the Pascal dynamic contour tonometer in monitoring intraocular pressure in unoperated eyes and eyes after LASIK. J Cataract Refract Surg 2004; 30: 746-751.

14 Doyle A, Lachkar Y. Comparison of dynamic contour tonometry with Goldman applanation tonometry over a wide range of central corneal thickness. I Glaucoma 2005; 14: 288-292.

15 Erickson DH, Goodwin D, Rollins M, Belaustegui A, Anderson C. Comparison of dynamic contour tonometry and Goldmann applanation tonometry and their relationship to corneal properties, refractive error, and ocular pulse amplitude. Optometry 2009; 80: 169-174.

16 Palmberg PF, Wiggs JL. Mechanisms of glaucoma. In: Janoff M, Duker JS (eds). Ophthalmology, 2nd ed. Mosby Co: St Louis, MO, USA, 2004, pp 1423-1430.

17 Shields MB, Ritch R, Krupin T. Intraocular pressure and tonometry. In: Ritch R, Shields MB, Krupin T (eds). The Glaucomas, 2nd ed. 2nd vol. Mosby, Times Mirror Company: St Louis, MO, USA, 1996, pp 1507-1520.
18 Unterlauft JD, Schadle N, Kasper K, Klink T, Geerling G. Comparison of dynamic contour tonometry and Goldmann applanation tonometry in keratoconus. Cornea 2011; 30(10): 1078-1082.

19 Bayer A, Sahin A, Hürmeriç A, Ozge G. Intraocular pressure values obtained by ocular response analyzer, dynamic contour tonometry, and Goldmann tonometry in keratokonic corneas. J Glaucoma 2010; 19: 540-545.

20 Goldich Y, Barkana Y, Avni I, Zadok D. Goldmann applanation tonometry versus ocular response analyzer for intraocular pressure measurements in keratoconic eyes. Cornea 2010; 29: 1011-1015.

21 Ozbek Z, Cohen EJ, Hammersmith KM, Rapuano CJ. Dynamic contur tonometry: a new way to asses intraocular pressure in ectatic corneas. Cornea 2006; 25: 890-894.

22 Alió JL, Shabayek MH. Corneal higher order aberrations: a method to grade keratoconus. J Refract Surg 2006; 22: 539-545.

23 Andreassen TT, Simonsen AH, Oxlund H. Biomechanical properties of keratoconus and normal corneas. Exp Eye Res 1980; 31: 435-441.

24 Kenney MC, Brown DJ. The cascade hypothesis of keratoconus. Contact Lens Anterior Eye 2003; 26: 139-146.

25 Browning AC, Bhan A, Rotchford AP, Shah S, Dua HS. The effect of corneal thickness on intraocular pressure measurement in patients with corneal pathology. Br J Ophthalmol 2004; 88: 1395-1399. 